\title{
Mapeamento das Diretrizes para Ensino de Computação na Educação Básica em uma Oficina de Banco de Dados
}

\author{
Bruna Oenning Amador \\ Universidade Tecnológica Federal do Paraná \\ Curitiba, PR, Brasil \\ brunaamador@alunos.utfpr.edu.br \\ Rita Cristina Galarraga Berardi \\ Universidade Tecnológica Federal do Paraná \\ Curitiba, PR, Brasil \\ rita@dainf.ct.utfpr.edu.br
}

\author{
Patricia Abe Turato \\ Universidade Tecnológica Federal do Paraná \\ Curitiba, PR, Brasil \\ patriciaturato@alunos.utfpr.edu.br \\ Sílvia Amélia Bim \\ Universidade Tecnológica Federal do Paraná \\ Curitiba, PR, Brasil \\ sabim@utfpr.edu.br
}

\begin{abstract}
The necessity of introducing the fundamental concepts of Computing in Brazilian elementary, middle and high school has been increasingly discussed by the Brazilian Computer Society (SBC). Nevertheless, to the best of our knowledge, there is no literature that explains how these concepts should be applied. This article describes how those specific Computing competences can be introduced in accordance with the guidelines defined by SBC. For this purpose, an analysis of a database workshop for high school girls was carried out, in order to map the guidelines to the workshop activities. This analysis serves as model for the creation of tools and courses in an articulated way with the guidelines of SBC, showing that existing materials can and should be harnessed.
\end{abstract}

\section{KEYWORDS}

Banco de Dados, Diretrizes da SBC, Educação Básica, Mulheres na Computação

\section{INTRODUÇÃO}

A Computação permite ampliar a compreensão sobre o mundo, além de aumentar a capacidade de aprendizagem e resolução de problemas, servindo como ferramenta de apoio ao aprendizado das demais disciplinas [SBC 2018a]. No entanto, para usar as ferramentas e tecnologias proporcionadas pela Computação, é necessário que seus fundamentos sejam compreendidos [SBC 2018b]. Levando isso em consideração, foram definidas, pela Sociedade Brasileira de Computação (SBC), as Diretrizes para Ensino de Computação na Educação Básica (DECEB). De acordo com Leila Ribeiro, diretora de Educação Básica da SBC, a finalidade da criação destas é mostrar quais habilidades e conceitos devem ser desenvolvidos em cada etapa do Ensino Fundamental e do Ensino Médio, servindo como guia às redes escolares [Ribeiro 2019].

Posto que as Diretrizes existem, a forma como o documento é apresentado acaba gerando incertezas sobre como aplicar o conteúdo na prática, uma vez que não existem instruções de como utilizá-las de forma adequada. Além disso, segundo [Ribeiro et al. $2020]^{1}$, o formato do material sobre os fundamentos da Computação e sobre os conteúdos que a SBC propõe que sejam ensinados na Educação Básica, atualmente, não é adequado para estudantes e

\footnotetext{
${ }^{1}$ Entre os minutos 42:27 e 42:48 do vídeo
}

docentes da Educação Básica. Sendo assim, é preciso gerar material alinhado com esses perfis, utilizando uma linguagem mais apropriada para o público, e trazer formas de executar o que foi proposto.

Considerando as DECEB, o presente artigo mostra o mapeamento destas a partir de uma oficina já ministrada e, consequentemente, busca facilitar a conexão de materiais novos ou existentes com as Diretrizes. A oficina escolhida foi a "Oficina de Banco de Dados com Aprendizado Cinestésico para Meninas do Ensino Médio” [Berardi et al. 2019], executada pelo projeto Emílias-Armações em Bits ${ }^{2}$ e o mapeamento foi realizado pelo projeto TIChers, ambos parceiros do Programa Meninas Digitais ${ }^{3}$ da SBC. Todas as informações a respeito do funcionamento da oficina e dos resultados obtidos a partir desta estão descritas no artigo [Berardi et al. 2019].

\section{MAPEAMENTO}

A oficina de Banco de Dados escolhida foi realizada em uma escola pública da cidade de Chapecó, no estado de Santa Catarina, e teve, como participantes, 43 meninas do $2^{\circ}$ e $3^{\circ}$ ano do Ensino Médio. O objetivo dessa oficina era motivar as estudantes a considerar a Computação como uma possível profissão e ensinar conceitos de Banco de Dados [Berardi et al. 2019]. Dessa forma, foram realizadas atividades, que foram divididas em quatro etapas principais: apresentação e provocação; dinâmica Human Branching Databases; apresentação dos conceitos de Banco de Dados; e teste de conhecimentos com a ferramenta Kahoot ${ }^{4}$.

Ao mapear as DECEB, foi possível identificar, nas atividades da oficina, a presença dos três eixos da Computação: Pensamento Computacional, o qual está ligado à resolução de problemas através da construção de algoritmos; Mundo Digital, que permite a codificação, o processamento e a distribuição de informação; e Cultura Digital, que busca promover a fluência no uso do conhecimento computacional [SBC 2018a].

$\mathrm{Na}$ apresentação, destacaram-se a presença de tecnologias no cotidiano e a influência dos avanços tecnológicos no surgimento de novas atividades profissionais. $\mathrm{Na}$ atividade de provocação, foram extraídas informações de um conjunto de dados não organizados e não estruturados disponibilizados em um quadro branco, a fim de refletir sobre a necessidade e a importância

\footnotetext{
${ }^{2}$ https://emilias.dainf.ct.utfpr.edu.br/

${ }^{3}$ http://meninas.sbc.org.br/

${ }^{4}$ https://bit.ly/2kCMSoM
} 
da utilização de Banco de Dados. Essas habilidades, trabalhadas na primeira etapa da oficina, estão relacionadas aos objetos de conhecimento: introdução à tecnologia digital, do eixo de Cultura Digital, sugerido para estudantes do primeiro ano do Ensino Fundamental; impactos da tecnologia digital, também do eixo de Cultura Digital, proposto para o Ensino Médio; e estruturas de dados estáticas, do eixo de Pensamento Computacional, recomendado ao quarto ano do Ensino Fundamental.

Na dinâmica Human Branching Databases, foram explorados os conceitos de organização em árvore e registros de tabelas. Segundo [Berardi et al. 2019], a montagem da árvore foi efetuada com perguntas sobre música, a fim de aumentar o interesse das adolescentes pela atividade e a tabela foi preenchida pelo monitor da oficina. Essa dinâmica proporcionou o conhecimento do conceito de árvore (eixo de Pensamento Computacional), habilidade proposta, nas Diretrizes , para estudantes do nono ano do Ensino Fundamental. Além disso, com essa dinâmica, as alunas puderam identificar a presença de tecnologia digital no cotidiano, devido à relação feita entre a atividade e os aplicativos de música, e puderam compreender a importância da organização dos dados. Dessa forma, as habilidades do eixo de Cultura Digital e de Pensamento Computacional trabalhadas durante a primeira etapa da oficina foram reforçadas.

$\mathrm{Na}$ terceira etapa, foi feita uma apresentação teórica dos conceitos de dado, informação e Banco de Dados, relacionando estes com as atividades realizadas anteriormente. Dessa forma, além das competências trabalhadas nas duas primeiras etapas da oficina, também foram abordadas, nesta etapa, algumas habilidades do eixo de Mundo Digital: a importância da descrição da informação e a necessidade de armazená-la e transmiti-la para a comunicação, sugeridas para o primeiro ano do Ensino Fundamental; e a relação entre informação e dado, proposta para estudantes do terceiro ano do Ensino Fundamental.

\section{DISCUSSÃO}

O domínio dos conceitos fundamentais da Computação permitirá que estudantes compreendam de forma mais completa o mundo e tenham, consequentemente, maior autonomia, flexibilidade, resiliência, pró-atividade e criatividade [SBC 2018b], sendo muito importante trabalhar esses conceitos desde cedo com os estudantes, para mostrar como a Computação é acessível a todos e descontruir preconceitos sobre a área.

A oficina analisada foi planejada e realizada com meninas do Ensino Médio que tiveram pouco ou nenhum contato prévio com conceitos de Computação, e obteve resultados positivos. No entanto, ao mapear as Diretrizes propostas pela SBC, foi identificado que a maioria dos conteúdos abordados nesta já poderiam ser trabalhados com turmas do Ensino Fundamental. Dessa forma, é possível adequá-la às Diretrizes adaptando a oficina de Banco de Dados para estudantes de menores faixas etárias.

A atividade realizada na segunda etapa da oficina [Berardi et al. 2019] foi baseada em uma dinâmica idealizada para crianças (a partir de 6 anos), a qual tinha como objetivo introduzir os fundamentos de Banco de Dados, utilizando o agrupamento das características físicas dos participantes em uma árvore binária [Dorling 2013]. Foram feitas, na oficina analisada, adaptações quanto à idade das pessoas envolvidas e quanto ao foco das perguntas utilizadas, trazendo músicas comuns para o público. Além disso, a dinâmica original não comtempla a questão da presença da tecnologia digital vista na adaptação por conta da analogia com os aplicativos de música. No entanto, esta habilidade do eixo da Cultura Digital também foi trabalhada na primeira etapa da oficina, deixando de ser necessária nessa atividade.

Portanto, ao invés da adaptação, seria possível utilizar o tema da dinâmica original, tornando a oficina, como um todo, viável para estudantes do Ensino Fundamental, de forma coerente às Diretrizes.

\section{CONSIDERAÇÕES FINAIS}

Uma importante contribuição da realização da oficina de Banco de Dados foi mostrar que oficinas lúdicas e cinestésicas possuem papel muito importante na divulgação da área de Computação, chamando a atenção das pessoas para outras características da área que não somente a programação [Berardi et al. 2019]. Como a oficina foi realizada com estudantes do Ensino Médio, segundo [Berardi et al. 2019], a maioria das participantes já mostrava preferência por alguma área específica e/ou já havia decidido qual curso seguir, não mudando a escolha da profissão. No entanto, a forma como a Computação, e seus diversos caminhos, é apresentada tem grande impacto para a desconstrução de vários pré-julgamentos. Dessa forma, ensinar as crianças desde cedo com essa visão fará com que mais meninas enxerguem a área de Computação como uma opção.

Além disso, a necessidade do ensino de Computação é comparável, atualmente, à da alfabetização no passado. Pessoas que não têm familiaridade com o poder da Computação não compreendem o mundo de hoje (e o do futuro) [SBC 2018b]. É visível, portanto, a importância de introduzir os conceitos de Computação desde os primeiros anos do Ensino Básico.

Para que isso seja viável, a seção "Discussão" deste artigo explicita a possibilidade de adaptação do formato de oficinas já idealizadas e realizadas de forma a contemplar as DECEB. Além disso, essa seção serve de modelo para a criação de ferramentas de modo articulado com as Diretrizes, mostrando que materiais existentes podem e devem ser aproveitados. No entanto, é fundamental esclarecer que a discussão presente neste artigo não excede o fato de que é preciso gerar material didático com uma linguagem mais apropriada para estudantes e docentes da Educação Básica [Ribeiro et al. 2020 $]^{5}$.

\section{REFERÊNCIAS}

SBC. Diretrizes para Ensino de Computação na Educação Básica, 2018a. URL https://www.sbc.org.br/educacao/diretrizes-para-ensino-de-computacao-naeducacao-basica. Acesso em: 02 dez. 2020.

SBC. Itinerário Formativo de Computação, 2018b. URL http://novoensinomedio.mec. gov.br/resources/downloads/pdf/Itinerário\%20Formativo\%20Computaç ao\%20$\% 20$ SBC.pdf. Acesso em: 02 dez. 2020.

Leila Ribeiro. Ensino de computação na educação básica: Diretrizes da sbc. Computação Brasil, (41):6-9, 2019. URL https://www.sbc.org.br/component/flippingbook/book/ 45. Acesso em: 03 dez. 2020

Leila Ribeiro, Ivan Claudio Pereira Siqueira, Lisandro Granville, and Maria Luciana Silva Nóbrega. Wei: Perspectivas e desafios do ensino de computação na educação básica, 2020. URL https://www.youtube.com/watch?v=9Qjytsjpyu8\&list= PLq1I6GEIwH6fuLF565vHp2EGm787tgpkI\&index=12. Acesso em: 10 dez. 2020.

Rita Berardi, Nádia Kozievitch, Silvia Bim, and Pedro Auceli. Oficina de banco de dados com aprendizado cinestésico para meninas do ensino médio. Anais do Workshop de Informática na Escola, 25(1):345-354, 2019. ISSN 2316-6541. doi: 10.5753/cbie.wie. 2019.345. URL https://www.br-ie.org/pub/index.php/wie/article/view/8521. Acesso em: 02 dez. 2020.

${ }^{5}$ Entre os minutos 57:37 e 57:48 do vídeo 
XII Computer on the Beach

7 a 9 de Abril de 2021, Online, SC, Brasil

Amador et al

Mark Dorling. Computing databases unplugged: Updated version. Digital Schoolhouse, 2013. URL https://classic.csunplugged.org/wp-content/uploads/2014/12

DatabasesUnplugged-Version2.pdf. Acesso em: 14 dez. 2020. 